Article

\title{
High Three-Dimensional Detection Accuracy in Piezoelectric-Based Touch Panel in Interactive Displays by Optimized Artificial Neural Networks
}

\author{
Shuo Gao ${ }^{1,2}$, Yanning Dai ${ }^{1}$, Vasileios Kitsos ${ }^{3}$ (D) Bo Wan ${ }^{4}$ and Xiaolei Qu ${ }^{1,2, *}$ \\ 1 School of Instrumentation and Optoelectronic Engineering, Beihang University, Beijing 100083, China; \\ shuo_gao@buaa.edu.cn (S.G.); 16171056@buaa.edu.cn (Y.D.) \\ 2 Beijing Advanced Innovation Center for Big Data-Based Precision Medicine, Beihang University, \\ Beijing 100083, China \\ 3 Electronic \& Electrical Engineering Department, University College London, London WC1E 7JE, UK; \\ kitsos.vasileios@gmail.com \\ 4 Cicada Canada Inc., Toronto, ON 15v1t7, Canada; sean.b.wan@gmail.com \\ * Correspondence: quxiaolei@buaa.edu.cn
}

Received: 7 January 2019; Accepted: 31 January 2019; Published: 13 February 2019

\begin{abstract}
High detection accuracy in piezoelectric-based force sensing in interactive displays has gained global attention. To achieve this, artificial neural networks (ANN)—successful and widely used machine learning algorithms-have been demonstrated to be potentially powerful tools, providing acceptable location detection accuracy of $95.2 \%$ and force level recognition of $93.3 \%$ in a previous study. While these values might be acceptable for conventional operations, e.g., opening a folder, they must be boosted for applications where intensive operations are performed. Furthermore, the relatively high computational cost reported prevents the popularity of ANN-based techniques in conventional artificial intelligence (AI) chip-free end-terminals. In this article, an ANN is designed and optimized for piezoelectric-based touch panels in interactive displays for the first time. The presented technique experimentally allows a conventional smart device to work smoothly with a high detection accuracy of above $97 \%$ for both location and force level detection with a low computational cost, thereby advancing the user experience, and serviced by piezoelectric-based touch interfaces in displays.
\end{abstract}

Keywords: piezoelectric-based touch panel; force sensing; detection accuracy; interactive displays; artificial neural network

\section{Introduction}

Touch-based interactivity has become a must-have function in smartphones and has created abundant applications in the last decade, improving users human-machine interactivity (HMI) in a convenient and highly efficient manner. The two-dimensional touch sensing, implemented by capacitive and resistive architectures, has traditionally dominated the market. However, the rapid development of information technology requires more data to be exchanged between the user and the end-terminal nowadays, boosting the popularity of three-dimensional force touch sensing.

Current force sensing in commercialized smartphones is supported by capacitive (e.g., iPhone 6S) and piezoresistive (e.g., iPhone X) techniques. The former integrates a layer of capacitive sensors into the backlight of the display to measure the distance shift due to the applied force between the cover glass and the backlight. In contrast, the latter utilizes the force-induced resistance change at the inserted electrodes to interpret the force level. To obtain the force sensing functionality without affecting the capacitive touch sensing, both the capacitive and the piezoresistive techniques require 
additional components, complex readout circuitry, and increased power consumption, all of which are highly undesirable. Hence, the demand for a simpler structured technique for passively detecting force information has been created. To that end, piezoelectric-based techniques have attracted attention for two main reasons: First, they can intrinsically convert mechanical stress into electrical energy, thus achieving force sensing passively. Secondly, they can be employed as the insulating layer of the original capacitive touch panel, obtaining three-dimensional touch sensing at the same time, without adding extra components.

The piezoelectric force touch panel prototypes have been broadly demonstrated [1-7]; however, their successful use in commercialized products has not yet been reported. This is due to the issue of non-uniformity of the over-panel force-voltage responsivity [8,9], which means that the same force strength will give rise to different voltage levels when the touch location varies. To address this issue, artificial neural networks (ANN), which are popular machine learning algorithms, are employed to map voltage levels to touch locations and force levels by training with labeled data. We previously developed an ANN-based technique to interpret the touch locations and force levels, and an acceptable detection accuracy (force detection: $93.3 \%$ and location detection: $95.2 \%$ ) was yielded [9]. Nevertheless, the detection accuracy is still expected to be boosted, since a 93.3\% detection accuracy cannot support complex software environments (e.g., action games), which demand intensive operations to be recognized precisely. Furthermore, the complexity of the developed technique strongly limits the technique's capabilities when implemented in artificial intelligence (AI) chip-free smartphones. Therefore, a modified technique for high detection accuracy in conventional chips is highly desirable, indicating that an optimization of the ANN structure is vital and urgent.

Since no research has yet been reported about the optimization of an ANN for piezoelectric-based force touch panels, in this paper, we investigate the relationship between the ANN hyperparameters (including hidden layer number, node number, cost functions, etc.) and the detection accuracy in terms of location and force level interpretation. We also study the effects of ANN structures (including hidden layer number and node number) on the computational cost, which is directly related to the processing time and power consumption of smart electronic devices and systems. The work presented here reveals the relationship between ANN structure, detection accuracy, and computational cost of the piezoelectric-based force touch panel for the first time, providing design considerations for both the academic and the industrial community.

This paper is organized as follows: Section 2 briefly reviews the working principle of piezoelectric-based force touch panels and their limitations when being used as interactive interfaces for smartphones; Section 3 explains the methodology; Section 4 demonstrates and discusses the experimental results.

\section{Literature Review}

A typical structured piezoelectric force touch panel is shown in Figure 1. It can be observed that three layers are used to construct the touch panel: A thin piezoelectric film layer in the middle for responding to force touch events and two electrode layers, one on top and one underneath the piezoelectric layer, for conveying the force-induced electric signals [10-15]. When a force touch is applied at the surface of the piezoelectric touch panel, the polarization of the piezoelectric layer becomes positive or negative according to the direction of the applied force, hence attracting the charges at the electrodes to form an electric potential. The relationship between the applied force and the induced polarization can be expressed as follows [16,17]:

$$
P_{i}=d_{i j} \sigma_{j k} \text { with } i, j, k=1,2,3,
$$

where $P_{i}$ is the induced polarization, and $\sigma_{j k}$ and $d_{i j k}$ denote the stress and piezoelectric coefficient, respectively. The coefficients remain the same for direct and inverse piezoelectric effects. The coefficients $d_{i j k}$ are symmetric in $j$ and $k$ [10]. Thus, Equation (1) can be simplified as follows: 


$$
P_{i}=d_{i j} \sigma_{j} \text { with } i=1,2,3 \text { and } j=1,2, \ldots, 6
$$

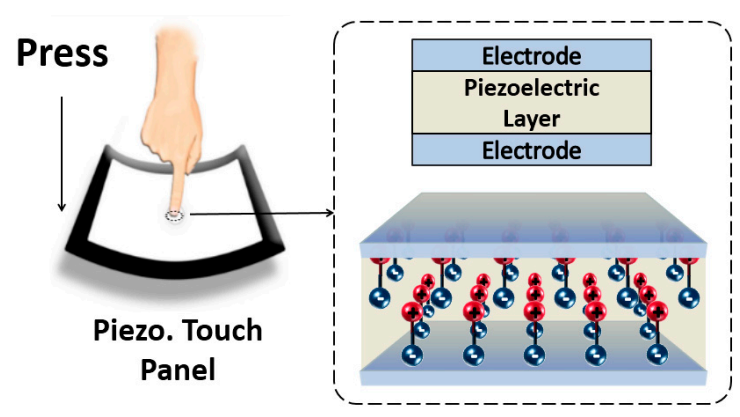

Figure 1. Conceptual depiction of a multi-functional stack-up.

From Equations (1) and (2), one can observe that the induced polarization is determined by both the piezoelectric coefficient and the stress vector. The piezoelectric coefficient does not change considerably after fabrication [18]. However, the same force touch can induce different stress vectors when applied at different locations of the touch panel, which is due to the mechanical properties and boundary conditions of the touch panel. As a result, the force-polarization responsivity is not consistent, which downgrades the detection accuracy $[8,9,19]$.

\section{Methodology}

In this section, the methodology used in this work is described. Details of the experimental setup and data acquisition technique are provided, followed by a description of the pre-processing method and the datasets for training and validation. Finally, the ANN for classification is presented.

\subsection{Experimental Setup and Data Acquisition}

The experiment is carried out with a previously developed multi-layered prototype utilized in Reference [19], consisting of a top cover layer, nine evenly distributed electrodes, a piezoelectric thin film layer, a ground electrode layer, and a bottom cover layer. Figure 2 depicts the topology, together with its geometries.

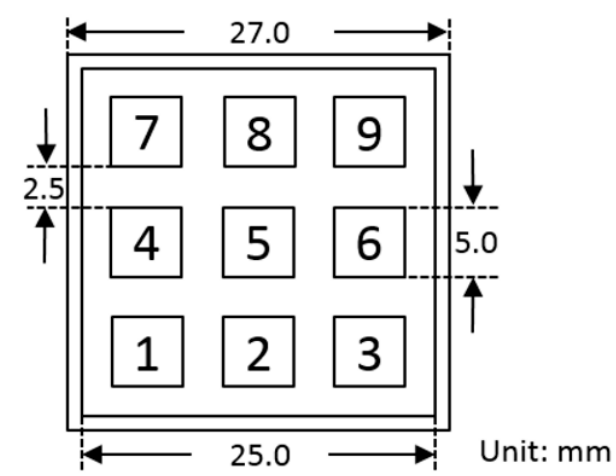

\begin{tabular}{|c|}
\hline Cover Glass: $0.5 \mathrm{~mm}$ \\
\hline Electrode: $0.1 \mu \mathrm{m}$ \\
\hline PVDF: $40 \mu \mathrm{m}$ \\
\hline Electrode: $0.1 \mu \mathrm{m}$ \\
\hline
\end{tabular}

Figure 2. Structure and geometry of the assembled touch panel.

Four volunteers to perform force touch events are employed for the experiment, and their physical body conditions are listed in Table 1. Each of them is required to tap 1350 times at nine specified locations (as shown in Figure 2) of the touch panel with three different force levels. Hence, a touch set is defined as tapping 50 times per location per force level. Because of the difference in physical body conditions and touch habits of the volunteers, the strength of the three force levels are highly dependent on the individual [19-21]. 
Table 1. Main characteristics of the four volunteers.

\begin{tabular}{ccccc}
\hline Volunteer No. & Gender & Height $\mathbf{( c m )}$ & Weight $\mathbf{( k g )}$ & Handedness \\
\hline 1 & Male & 173 & 71 & Right \\
2 & Male & 182 & 90.5 & Right \\
3 & Female & 167 & 65 & Left \\
4 & Female & 159 & 44 & Right \\
\hline
\end{tabular}

The voltages across the nine piezoelectric-based capacitors are continuously monitored. The retrieved raw data for one touch subset are given in Figure 3 as an example, including the raw data from the nine channels; each channel illustrates the relationship between voltage and time. Each peak in the raw data indicates a force touch event. Since the force-induced stress at one location can propagate to other areas, all nine channels produce an output signal when a touch event occurs. However, the amplitudes of the signals are different and depend on the stress distribution on the touch panel. Furthermore, as a complete force touch event consists of "press" and "release" actions, the polarizations of the piezoelectric layer change twice, resulting in positive and negative peaks, as illustrated in Figure 3. The "press" action induces a positive electric signal and is considered to represent the force level of the force touch event.

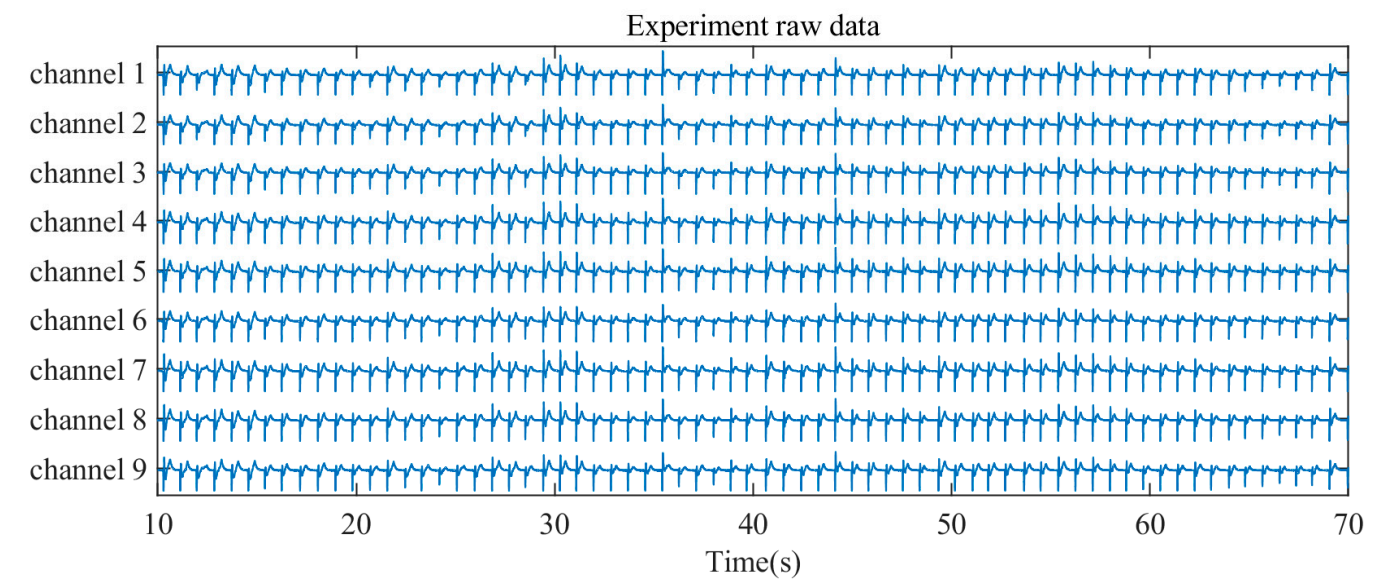

Figure 3. Experimental raw signal data.

\subsection{Pre-Processing and Dataset Preparation}

To obtain the information carried in the press-induced electric signals, a pre-processing algorithm has been developed through four steps to extract the voltage peaks from each channel, as shown in Figure 4. First, the direct current (DC) offset of each channel is removed by subtracting the mean voltage of each channel. Second, envelope detection is conducted to each channel by the Hilbert Transform method, as the voltage variations are both positive and negative. Third, we assume that the voltage variation peaks of different channels appear at the same time and calculate the mean value of the nine different channels to suppress random noise. Finally, the voltage peaks are detected by finding the local maximum voltage values. After the voltage peaks for each channel have been acquired, two ANNs are employed to classify locations and force levels. Their details will be given in the next sub-section.

After pre-processing, nine voltage peaks for each force touch event are obtained and used as features to classify the touch locations and force levels. As mentioned before, 50 touches are performed at each location at the same force amplitude, so 1350 touch signals are obtained in total from each volunteer. Since one touch provides one output data point (including nine peak values from all nine channels), the dataset for a single volunteer consists of 50.9.3 output data points. Then, for the training, validation, and testing of the ANN, the dataset is further separated into a training set, a validation set, and a test set. The training set has 40 force touch events for each location and each force level; hence, it 
has $40 \cdot 9 \cdot 3$ data points, which makes up $80 \%$ of the data; the validation and test sets have 10 events each (5.9.3 data points, which makes up $10 \%$ each).

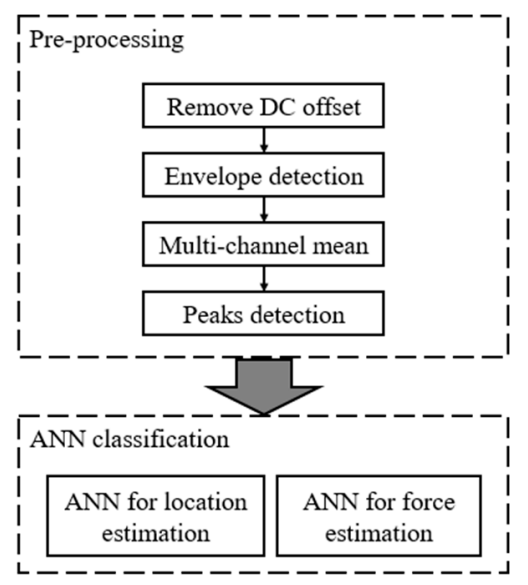

Figure 4. Flow chart for the data pre-processing and classification.

\subsection{Pre-Processing Multi-Layers Neural Networks for Classification}

Convolution neural networks (CNN) [22-29] and recurrent neural networks (RNN) [30-35] are conventionally used to extract features for image and audio applications, and a fully connected network [36-38] can be used as an output layer of a CNN or RNN. However, a fully connected network can also be used alone for classification. In our case, the signal features have already been extracted with the help of the pre-processing step explained above, and, thus, we only employed the fully connected network for location and force level classification.

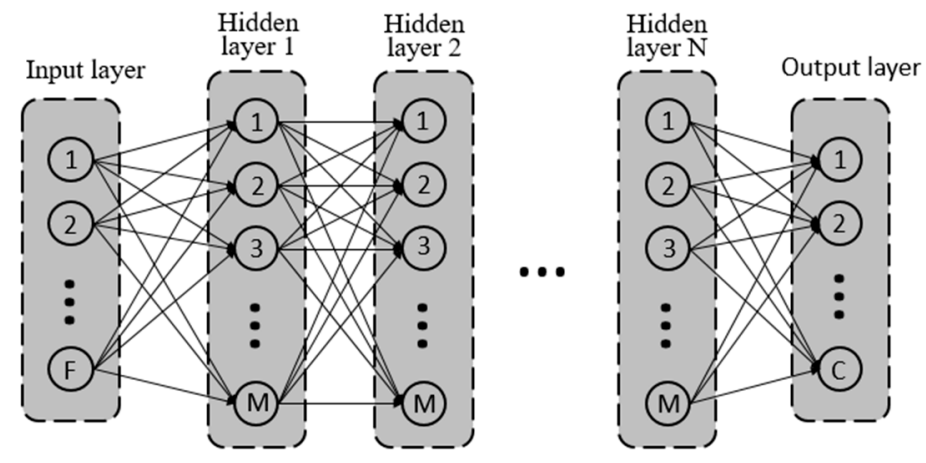

Figure 5. Schematic diagram of a fully connected artificial neural network (ANN). F is the number of features (nine for both force level and location classification); $\mathrm{N}$ and $\mathrm{M}$ are the hidden layer number and node number; $\mathrm{C}$ is the number of object classes (three for force level classification and nine for location classification). Note: Each hidden layer and output layer also have a bias input, which is ignored in this figure.

The fully connected ANN has three kinds of layers with multiple nodes embedded, including an input layer, an output layer, and multiple hidden layers (Figure 5). The number of the input and output layers' nodes are identical to the number of features and object classes, which are nine and nine for location classification, and nine and three, respectively, for force level classification. Alternatively, the number of hidden layers and their embedded nodes are two independent hyperparameters, which have a significant effect on variance and computational complexity. In general, an increase in the value of these two numbers will have a negative effect on the computational cost; however, ANN becomes more powerful to deal with complex classification scenarios. Thus, the tradeoff between the performance and computational cost must be taken into careful account when designing a fully connected ANN. 
The computational time cost includes training time and classification time costs, both of which increase as the layer number and node number increase. The training time cost only occurs once, and that is during the initial training of the ANN. However, the classification time cost occurs every time the user presses the touch panel. Therefore, a high tolerance is usually set for the training time cost, and a low tolerance for the classification time cost. In this study, the training time cost is defined experimentally, while the classification time cost is analyzed theoretically. We implemented our ANNs with different layer numbers and node numbers using Keras. Regarding the training time cost, we trained the ANNs using a laptop (Lenovo ThinkPad X1) with a CPU (Intel(R) Core(TM) i5-3427U @ $1.8 \mathrm{GHz}$ ), and the time cost of training was recorded. With regard to the classification time cost, it is positively correlated to the number $(\mathrm{W})$ of total weight parameters, which can be calculated by:

$$
W=(F+C) M+(N-1) M^{2}, \quad(N \geq 1),
$$

where $F$ is the feature number (nine for both force level and location classification), $M$ and $N$ are the node number and layer number, respectively, and $C$ is the number of object classes.

The cost function used for training can also greatly affect the performance [39-44]. Three commonly used cost functions have been chosen: The mean-squared-error loss function, the categorical cross-entropy loss function, and the binary cross-entropy loss function, and their classification accuracy was compared.

For the minimization of the loss function during the training process, a stochastic gradient-based optimizer (Adam 2015 [45]) is used to optimize the values of weights and bias of the network, due to its computational efficiency and its low memory requirements [46]. The active function used for the input and hidden layers is 'ReLU', since it has high time efficiency $[47,48]$. In order to avoid overfitting, we make use of L2 regularization with a regularization factor of 0.001 , which is selected experimentally [49]. The learning rate is experimentally selected as 0.0005 .

\section{Results and Discussion}

The pre-processing results after each step are plotted in Figure 6. One can observe that the DC offset at the original output signal is about $0.8 \mathrm{~V}$ (Figure 6a). After successfully removing the DC offset, the mean of the signal is close to $0 \mathrm{~V}$ (Figure $6 \mathrm{~b}$ ). The envelope detection algorithm is then applied to the offset-free signal, since there are positive and negative components during a force touch event (Figure 6c). Based on the assumption that voltage variation peaks of different channels appear at the same time, the mean value of the nine channels is calculated, as Figure $6 \mathrm{~d}$ depicts. Finally, the peaks are located and marked by circles in the detected peaks figure. As Figure 6e confirms, the proposed pre-processing method is capable of appropriately detecting the peaks of the signal. Figure 7 shows the response voltages from one volunteer at the same location with different force levels. As shown, our prototype touch panel has different ranges of response voltage for different force levels.

After the pre-processing, the training of the force level classification ANN is performed using three different cost functions, the mean square error cost function, the categorical cross-entropy cost function, and the binary cross-entropy cost function. The corresponding accuracy and loss results are provided in Figure 8. Based on this figure, one can not only observe that the accuracies of both the training set and the validation set are identical, but also that their losses are close to each other. Hence, it is demonstrated that there is no obvious overfitting in this training. Among these three cost functions, the binary cross-entropy cost function provides the best accuracy $(98.5 \%)$, hence, it is chosen as the cost function for force level classification. 


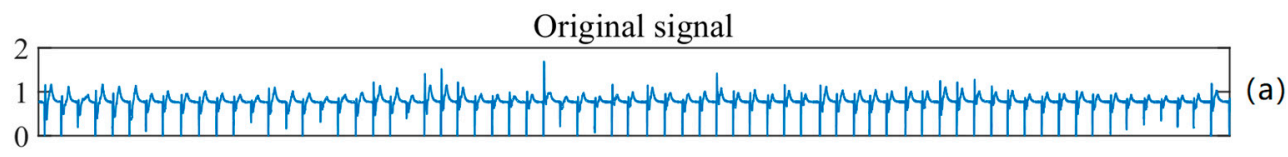
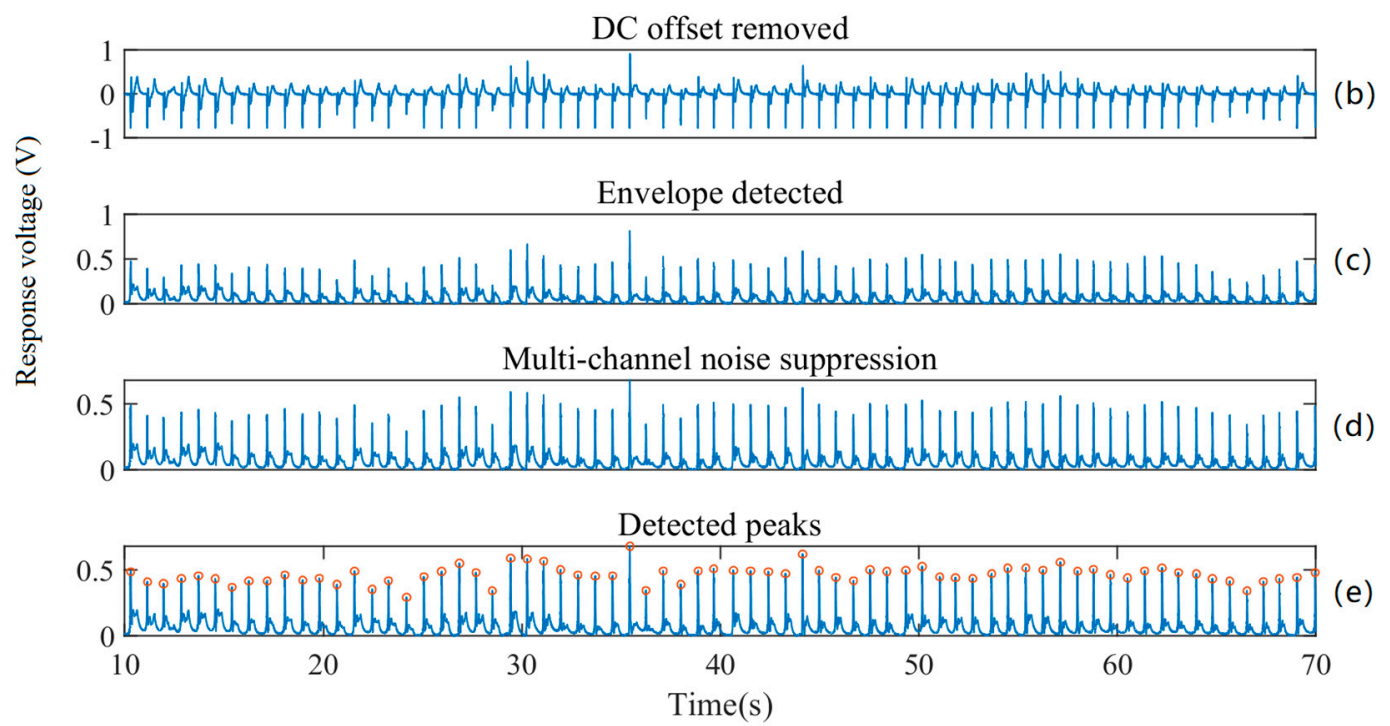

Figure 6. Signal pre-processing results: (a) original signal; (b) DC offset removed signal; (c) signal after envelope detection; (d) signal after multi-channel noise suppression; (e) detected signal peaks.

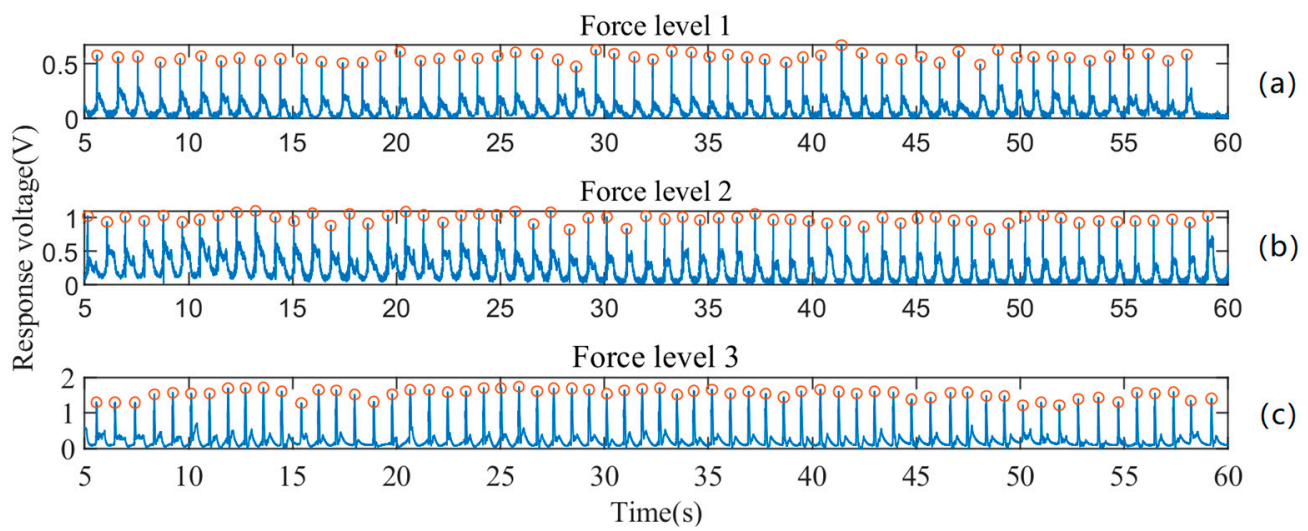

Figure 7. Response voltages at the same location with different force levels; (a), (b) and (c): response voltages of force level 1,2 and 3.

The three cost functions used above are also applied for training of the location classification ANN, and the results are shown in Figure 9. Unlike force level classification, over-fitting appears in two of the three cost functions: The mean-squared error function and categorical cross-entropy cost function. The over-fittings can be seen by observing that the accuracies of the training sets are higher than those of the validation sets. In contrast, the accuracy of the binary cross-entropy cost function for the training set is close to that of the validation set (Figure 9e). In fact, it is $97.8 \%$ higher than that of their counterparts. Therefore, the binary cross-entropy cost function is selected for location classification. 

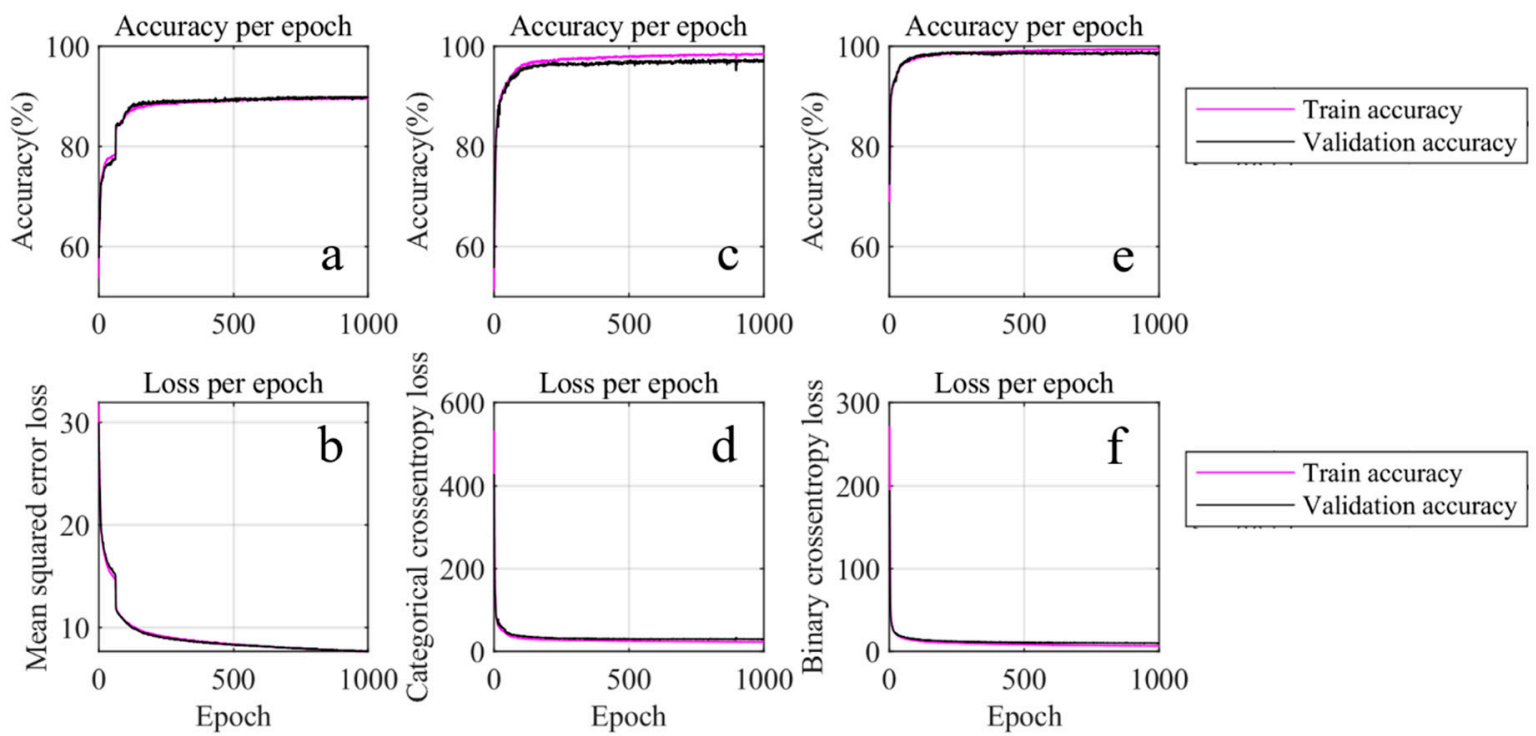

Figure 8. Force level estimation using different loss functions. (a,c,e) are the accuracy during training using the mean-squared error loss, categorical cross-entropy loss, and binary cross-entropy loss, respectively. $(\mathbf{b}, \mathbf{d}, \mathbf{f})$ are the loss of $(\mathbf{a}, \mathbf{c}, \mathbf{e})$.
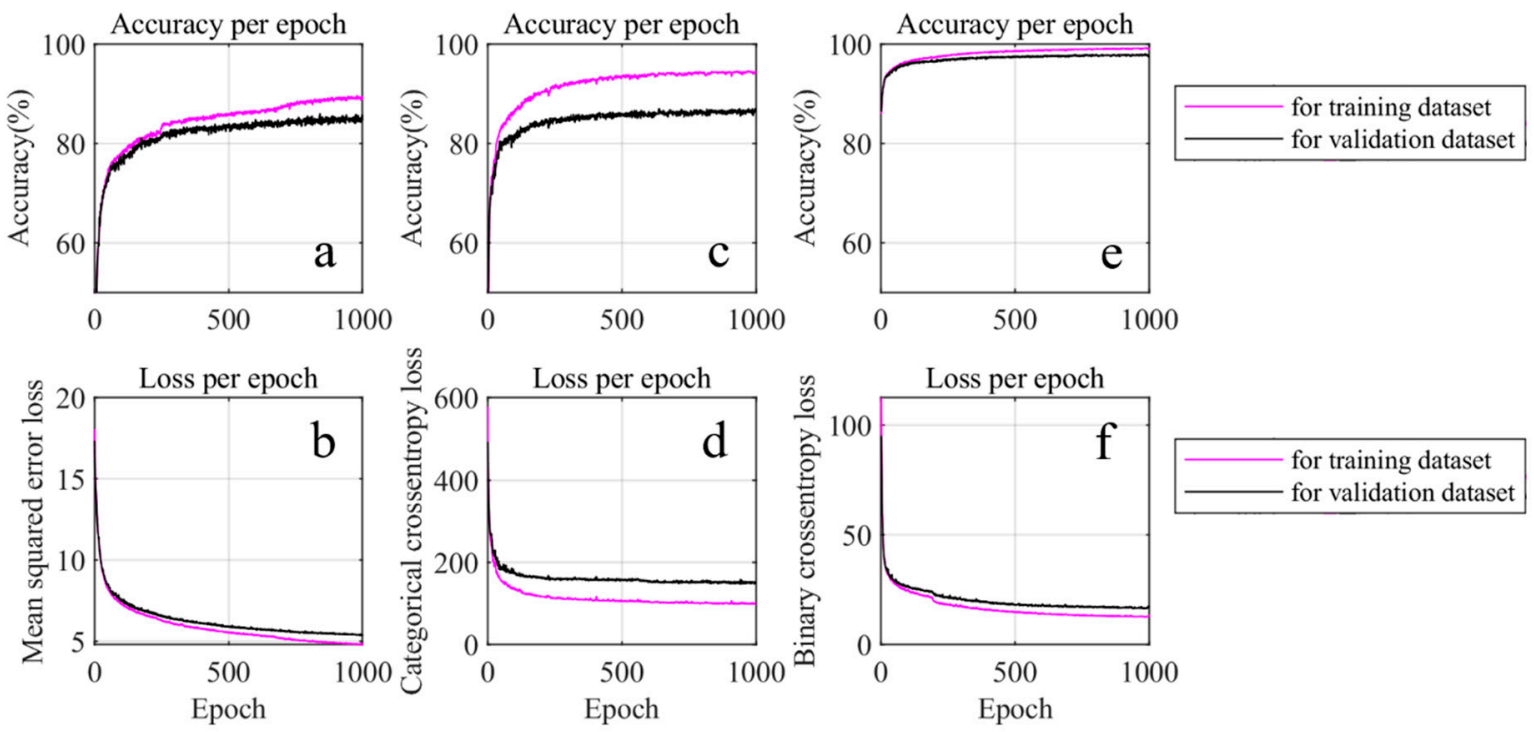

Figure 9. Location estimation using different cost functions. (a,c,e) are accuracy during training using mean-squared error loss, categorical cross-entropy loss, and binary cross-entropy loss, respectively. $(\mathbf{b}, \mathbf{d}, \mathbf{f})$ are the loss of $(\mathbf{a}, \mathbf{c}, \mathbf{e})$.

Figure 10 shows the correlation between the achieved accuracy and the fully connected ANN structure for different layer numbers and node numbers. Figure 10a shows the accuracy of force level classification. For layer numbers 1 to 3 and node numbers 4 to 32 , the accuracy increases as the layer number or node number increases. The reason for this is that the ANN structure becomes more complex, hence, it can fit complex classification functions. However, when the layer number becomes larger than 3, and the node number greater than 32, no significant change in the accuracy is observed. Therefore, a layer number of 3 and a node number of 32 are selected for the force level classification in this paper. Figure $10 \mathrm{~b}$ shows the accuracy of the location classification, which is slightly lower than that of the force level classification. As the layer number increases from 1 to 4 and the node number from 4 to 64 , the accuracy also increases. However, for a layer number larger than 3 and a node number larger than 32, the accuracy does not change significantly. Thus, a layer number of 3 and 
a node number of 64 are chosen for location classification in this paper. Then, the accuracy of the test set is calculated as $97.7 \%$ and $97.0 \%$ for force and location classification, respectively.
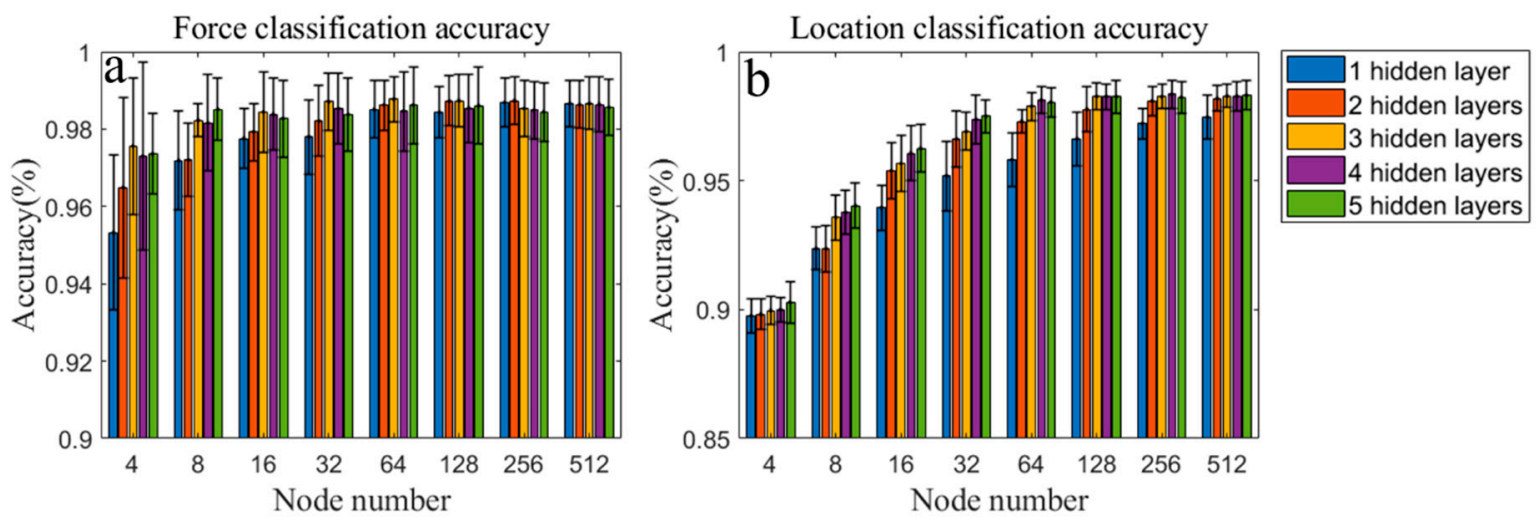

Figure 10. Classification accuracy of ANNs using different layer numbers and node numbers. (a): accuracy of force claasification; (b): accuracy of location classification.

The training time costs for location and force level classification ANNs are given in Figure 11. The time cost remarkably increases when the node number is greater than 64 . Also, it rises as the layer number increases. When the ANN has 5 hidden layers and 512 nodes, the maximum training time cost for location and force level classification are 418.2 and $424.8 \mathrm{~ms} /$ epoch, respectively.

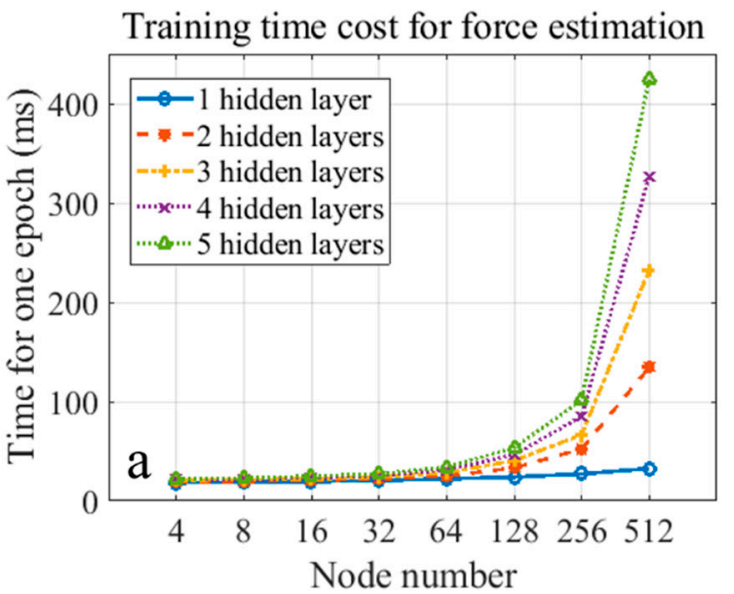

(a)
Training time cost for location estimation

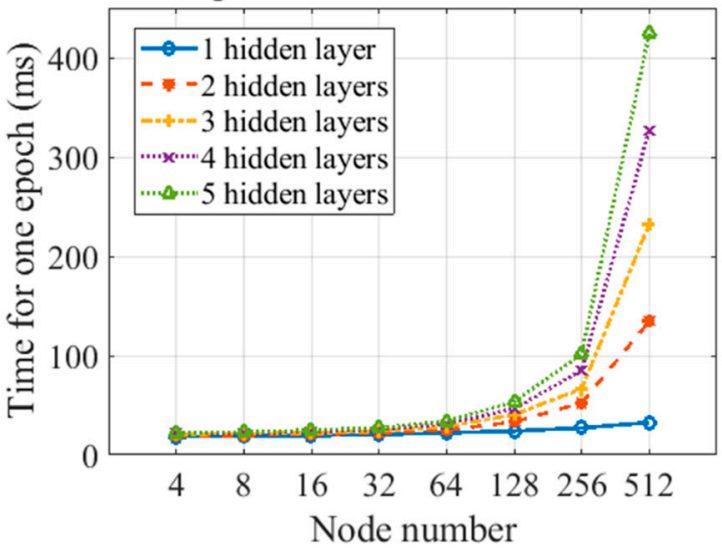

(b)

Figure 11. Training time cost for (a) force estimation and (b) location estimation.

Figure 12 gives the parameter number for force classification ANN, which is shown on a logarithmic scale, since it grows exponentially. The parameter number for the location classification ANN is a little larger than that of the force level classification ANN, because the output layer node number increases from three-nine. The classification time cost increases as the parameter number increases. Thus, the classification cost also grows exponentially. The location classification time measured for one touch is $0.98 \mathrm{~ms}$ for an ANN with 5 hidden layers and 512 nodes for each layer. 


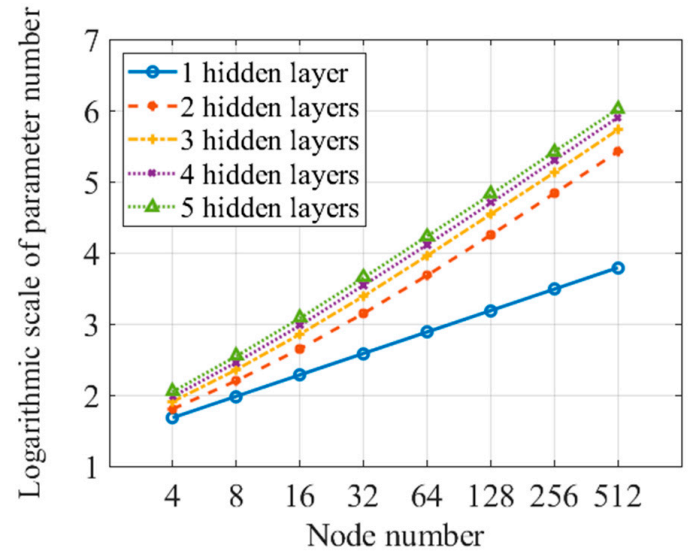

Figure 12. Logarithmic scale of parameter number for force classification ANN.

\section{Conclusions}

ANNs have been used in the literature to address the inconsistent force-voltage responsivity issue in piezoelectric-based force touch panels for interactive displays. However, the high computational complexity and the relatively low detection accuracy limit their successful use in conventional consumer products. The work presented in this paper bridges this gap by investigating the relationship between the ANN hyperparameters, detection accuracy, and computational cost. After carefully designing the ANN on the basis of the specific characteristics of the piezoelectric-based touch events, both a high detection accuracy of above $97 \%$ and a low computational cost of less than 0.98 ms have been achieved experimentally.

Author Contributions: Conceptualization, S.G. and X.Q.; methodology, S.G., Y.D. and X.Q.; validation, S.G., Y.D., X.Q., V.K. and B.W.; formal analysis, X.Q.; investigation, S.G.; resources, X.Q.; data curation, S.G. and X.Q.; Writing-Original Draft preparation, S.G., Y.D. and X.Q.; Writing-Review and Editing, S.G., Y.D. and V.K.; visualization, X.Q. and B.W.

Funding: This research was funded by the National Natural Science Foundation, grant number $61803017,61827802$.

Acknowledgments: The authors thank Cambridge Touch Technologies (Cambridge, UK) for its generous provision of the touch panel stack-up and readout circuit architectures.

Conflicts of Interest: The authors declare no conflict of interest.

\section{References}

1. Gao, S.; Wu, X.; Ma, H.; Robertson, J.; Nathan, A. Ultrathin Multi-functional Graphene-pvdf Layers for Multi-dimensional Touch Interactivity for Flexible Displays. ACS Appl. Mater. Interfaces 2017, 9, 18410-18416. [CrossRef] [PubMed]

2. Gao, S.; Nathan, A. P-180: Force Sensing Technique for Capacitive Touch Panel. In SID Symposium Digest of Technical Papers; Wiley: Hoboken, NJ, USA, 2016; Volume 47, pp. 1814-1817.

3. Vuorinen, T.; Zakrzewski, M.; Rajala, S.; Lupo, D.; Vanhala, J.; Palovuori, K. Printable, Transparent, and Flexible Touch Panels Working in Sunlight and Moist Environments. Adv. Funct. Mater. 2015, 24, 6340-6347. [CrossRef]

4. Park, W.; Yang, J.H.; Kang, C.G.; Lee, Y.G.; Hwang, H.J.; Cho, C. Characteristics of a Pressure Sensitive Touch Sensor Using a Piezoelectric pvdf-trfe/mos Stack. Nanotechnology 2013, 24, 475501. [CrossRef] [PubMed]

5. Bae, S.H.; Kahya, O.; Sharma, B.K.; Kwon, J.; Cho, H.J.; Ozyilmaz, B.; Ahn, J.H. Graphene-P (VDF-TrFE) Multilayer Film for Flexible Applications. ACS Nano 2013, 7, 3130-3138. [CrossRef] [PubMed]

6. Reynolds, K.; Petr, S.; Arnulf, G. Invited Paper: Touch and Display Integration with Force. In SID Symposium Digest of Technical Papers; Wiley: Hoboken, NJ, USA, 2016; Volume 47, pp. 617-620.

7. Chu, X.; Liu, J.; Gao, R.; Chang, J.; Li, L. Design and Analysis of a Piezoelectric Material Based Touch Screen with Additional Pressure and Its Acceleration Measurement Functions. Smart Mater. Struct. 2013, 22, 125008. [CrossRef] 
8. Gao, S.; Wu, L. Why Piezoelectric Based Force Sensing is not Successful in Interactive Displays? IEEE Consum. Electron. Mag. 2019, 8, in press.

9. Gao, S.; Duan, J.; Kitsos, V.; Selviah, D.R.; Nathan, A. User-Oriented Piezoelectric Force Sensing and Artificial Neural Networks in Interactive Displays. IEEE J. Electron. Device Soc. 2018, 6, 766-773. [CrossRef]

10. Gao, S.; Arcos, V.; Nathan, A. Piezoelectric vs. Capacitive Based Force Sensing in Capacitive Touch Panels. IEEE Access 2016, 4, 3769-3774. [CrossRef]

11. Manbachi, A.; Cobbold, R.S.C. Development and Application of Piezoelectric Materials for Ultrasound Generation and Detection. Ultrasound 2011, 19, 187-196. [CrossRef]

12. Nathan, A.; Henry, B. Microtransducer CAD: Physical and Computational Aspects (Computational Microelectronics); Springer: Vienna, Austria, 1999.

13. Maseeh, F.; Schmidt, M.A.; Allen, M.G.; Senturia, S.D. Calibrated Measurements of Elastic Limit, Modulus, and the Residual Stress of Thin Films Using Micromachined Suspended Structures. In Proceedings of the IEEE Solid-State Sens and Actuator Workshop, Hilton Head Island, SC, USA, 6-9 June 1988.

14. Mohammadi, B.; Yousefi, A.A.; Bellah, S.M. Effect of Tensile Strain Rate and Elongation on Crystalline Structure and Piezoelectric Properties of PVDF Thin Films. Polym. Test. 2007, 26, 42-50. [CrossRef]

15. Saketi, P.; Latifi, S.K.; Hirvonen, J.; Rajala, S.; Vehkaoja, A.; Salpavaara, T.; Lekkala, J.; Kallio, P. PVDF Microforce Sensor for the Measurement of Z-directional Strength in Paper Fiber Bonds. Sens. Actuators A Phys. 2015, 222, 194-203. [CrossRef]

16. Cain, M.G. (Ed.) Characterisation of Ferroelectric Bulk Materials and Thin Films, 2nd ed.; Springer: Dordrecht, The Netherlands, 2014.

17. Dineva, P.; Gross, D.; Müller, R.; Rangelov, T. Dynamic Fracture of Piezoelectric Materials, Solid Mechanics and Its Applications; Springer International Publishing: Cham, Switzerland, 2014.

18. Gao, S. A Multifunctional Touch Interface for Multidimensional Sensing. Ph.D. Thesis, University of Cambridge, Cambridge, UK, 2018.

19. Gao, S.; Nathan, A. P-209: Augmenting Capacitive Touch with Piezoelectric Force Sensing. In SID Symposium Digest of Technical Papers; Wiley: Hoboken, NJ, USA, 2017; Volume 48, pp. 2068-2071.

20. Jones, L.A. Perception and Control of Finger Forces. In Proceedings of the ASME Dynamic Systems and Control Division, Anaheim, CA, USA, 15-20 November 1998; Volume 5, pp. 133-137.

21. Jones, L.A. The Control and Perception of Finger Forces. In The Human Hand as an Inspiration for Robot Hand Development; Springer: Cham, Switzerland, 2014.

22. Chen, L.; Papandreou, G.; Kokkinos, I.; Murphy, K.; Yuille, A.L. DeepLab: Semantic Image Segmentation with Deep Convolutional Nets, Atrous Convolution, and Fully Connected CRFs. IEEE Trans. Pattern Anal. Mach. Intell. 2018, 40, 834-848. [CrossRef] [PubMed]

23. Kim, Y. Convolutional Neural Networks for Sentence Classification. In Proceedings of the Conference on Empirical Methods in Natural Language Processing, Doha, Qatar, 25-29 October 2014; pp. 1746-1751.

24. Krizhevsky, A.; Sutskever, I.; Hinton, G.E. ImageNet Classification with Deep Convolutional Neural Networks. In Proceedings of the 25th International Conference on Neural Information Processing Systems, Lake Tahoe, NV, USA, 3-6 December 2012; pp. 1097-1105.

25. Long, J.; Shelhamer, E.; Darrell, T. Fully Convolutional Networks for Semantic Segmentation. In Proceedings of the IEEE Conference on Computer Vision and Pattern Recognition, Boston, MA, USA, 7-12 June 2015; pp. 3431-3440.

26. Ronneberger, O.; Fischer, P.; Brox, T. U-Net: Convolutional Networks for Biomedical Image Segmentation. In Proceedings of the International Conference on Medical Image Computing and Computer-Assisted Intervention, Munich, Germany, 5-9 October 2015; pp. 234-241.

27. Shelhamer, E.; Long, J.; Darrell, T. Fully Convolutional Networks for Semantic Segmentation. IEEE Trans. Pattern Anal. Mach. Intell. 2017, 39, 640-651. [CrossRef] [PubMed]

28. Simonyan, K.; Zisserman, A. Very Deep Convolutional Networks for Large-Scale Image Recognition. In Proceedings of the International Conference on Learning Representations, San Diego, CA, USA, 7-9 May 2015.

29. Szegedy, C.; Liu, W.; Jia, Y.; Sermanet, P.; Reed, S.; Anguelov, D.; Erhan, D.; Vanhoucke, V.; Rabinovich, A. Going Deeper with Convolutions. In Proceedings of the 2015 IEEE Conference on Computer Vision and Pattern Recognition (CVPR), Boston, MA, USA, 7-12 June 2015; pp. 1-9. 
30. Bengio, Y.; Simard, P.Y.; Frasconi, P. Learning Long-term Dependencies with Gradient Descent is Difficult. IEEE Trans. Neural Netw. 1994, 5, 157-166. [CrossRef] [PubMed]

31. Graves, A.; Jaitly, N. Towards End-To-End Speech Recognition with Recurrent Neural Networks. In Proceedings of the International Conference on Machine Learning, Beijing, China, 21-26 June 2014; pp. 1764-1772.

32. Graves, A.; Mohamed, A.; Hinton, G.E. Speech Recognition with Deep Recurrent Neural Networks. In Proceedings of the International Conference on Acoustics, Speech, and Signal Processing, Vancouver, BC, Canada, 26-30 May 2013; pp. 6645-6649.

33. Osako, K.; Singh, R.; Raj, B. Complex Recurrent Neural Networks for Denoising Speech Signals. In Proceedings of the Workshop on Applications of Signal Processing to Audio and Acoustics, New Paltz, NY, USA, 18-21 October 2015; pp. 1-5.

34. Sak, H.; Senior, A.W.; Rao, K.; Beaufays, F. Fast and Accurate Recurrent Neural Network Acoustic Models for Speech Recognition. In Proceedings of the Conference on International Speech Communication Association, Dresden, Germany, 20 May 2015; pp. 1468-1472.

35. Williams, R.J.; Zipser, D. A Learning Algorithm for Continually Running Fully Recurrent Neural Networks. Neural Comput. 1989, 1, 270-280. [CrossRef]

36. Snyder, M.M.; Ferry, D.K. Open Loop Stability Criterion for Layered and Fully-connected Neural Networks. Neural Netw. 1988, 1, 133.

37. Wan, L.; Zeiler, M.D.; Zhang, S.; Cun, Y.L.; Fergus, R. Regularization of Neural Networks using DropConnect. In Proceedings of the International Conference on Machine Learning, Atlanta, GA, USA, 16-21 June 2013; pp. 1058-1066.

38. Xu, Q.; Zhang, M.; Gu, Z.; Pan, G. Overfitting Remedy by Sparsifying Regularization on Fully-connected Layers of CNNs. Neurocomputing 2018, 328, 69-74. [CrossRef]

39. Gajowniczek, K.; Chmielewski, L.J.; Orlowski, A.; Ząbkowski, T. Generalized Entropy Cost Function in Neural Networks. In Proceedings of the International Conference on Artificial Neural Networks, Alghero, Italy, 11-14 September 2017; pp. 128-136.

40. Hampshire, J.B.; Waibel, A. A Novel Objective Function for Improved Phoneme Recognition Using Time-delay Neural Networks. IEEE Trans. Neural Netw. 1990, 1, 216-228. [CrossRef]

41. Janocha, K.; Czarnecki, W.M. On Loss Functions for Deep Neural Networks in Classification. Schedae Inform. 2017, 2016, 4959. [CrossRef]

42. Nasr, G.E.; Badr, E.A.; Joun, C. Cross Entropy Error Function in Neural Networks: Forecasting Gasoline Demand; The Florida AI Research Society: Melbourne, FL, USA, 2002; pp. 381-384.

43. Xu, L. Original Contribution: Least Mean Square Error Reconstruction Principle for Self-organizing Neural-nets. Neural Netw. 1993, 6, 627-648. [CrossRef]

44. Zhang, Z.; Sabuncu, M.R. Generalized Cross Entropy Loss for Training Deep Neural Networks with Noisy Labels. In Proceedings of the Neural Information Processing Systems, Montreal, NU, Canada, 3-8 December 2018.

45. Kingma, D.P.; Ba, J. Adam: A Method for Stochastic Optimization. In Proceedings of the International Conference on Learning Representations, San Diego, CA, USA, 7-9 May 2015.

46. Andrychowicz, M.; Denil, M.; Gomez, S.; Hoffman, M.W.; Pfau, D.; Schaul, T.; Shillingford, B.; De Freitas, N. Learning to Learn by Gradient Descent by Gradient Descent. In Proceedings of the Advances in Neural Information Processing Systems 29 (NIPS 2016), Barcelona, Spain, 5-10 December 2016; pp. 3981-3989.

47. Li, Y.; Yuan, Y. Convergence Analysis of Two-layer Neural Networks with ReLU Activation. In Proceedings of the Neural Information Processing Systems, Long Beach, CA, USA, 4-9 December 2017; pp. 597-607.

48. Nair, V.; Hinton, G.E. Rectified Linear Units Improve Restricted Boltzmann Machines. In Proceedings of the International Conference on Machine Learning, Haifa, Israel, 21-24 June 2010; pp. 807-814.

49. Girosi, F.; Jones, M.J.; Poggio, T. Regularization Theory and Neural Networks Architectures. Neural Comput. 1995, 7, 219-269. [CrossRef]

(C) 2019 by the authors. Licensee MDPI, Basel, Switzerland. This article is an open access article distributed under the terms and conditions of the Creative Commons Attribution (CC BY) license (http:/ / creativecommons.org/licenses/by/4.0/). 